\title{
Research Square \\ Quantification of Vortex Flow in Pulmonary Arteries of Patients With Chronic Thromboembolic Pulmonary Hypertension
}

Hiroki Kamada ( $\nabla$ hkamada@rad.med.tohoku.ac.jp )

Tohoku University Hospital: Tohoku Daigaku Byoin https://orcid.org/0000-0003-3341-9396

Hideki Ota

Tohoku University Hospital: Tohoku Daigaku Byoin

Masanori Nakamura

Nagoya Institute of Technology Electrical and Mechanical Engineering: Nagoya Kogyo Daigaku Denki

Kikai Kogakuka Denki Kikai Kogaku Kenkyuka

Wenyu Sun

Tohoku University Hospital: Tohoku Daigaku Byoin

\section{Tatsuo Aoki}

Tohoku University: Tohoku Daigaku

Haruka Sato

Tohoku University: Tohoku Daigaku

Koichiro Sugimura

Tohoku University: Tohoku Daigaku

\section{Kei Takase}

Tohoku University Hospital: Tohoku Daigaku Byoin

\section{Research Article}

Keywords: 4D-flow MRI, CTEPH, BPA, blood flow

Posted Date: April 21st, 2021

DOI: https://doi.org/10.21203/rs.3.rs-436343/v1

License: (9) This work is licensed under a Creative Commons Attribution 4.0 International License.

Read Full License

Version of Record: A version of this preprint was published at European Journal of Radiology on January 1st, 2022. See the published version at https://doi.org/10.1016/j.ejrad.2021.110142. 
1 Quantification of vortex flow in pulmonary arteries of patients with chronic

2 thromboembolic pulmonary hypertension

3

4 Purpose: This study proposes an objective method of quantifying the vortex flow in

5 pulmonary arteries to compare the duration of its presence before and after balloon

6 pulmonary angioplasty (BPA) in patients with chronic thromboembolic pulmonary

7 hypertension (CTEPH).

8

9 Methods: Thoracic 4D-flow magnetic resonance imaging was performed in $28 \mathrm{CTEPH}$

10 patients before and after BPA. Planes were set in pulmonary arteries to evaluate volume

11 flow rate (VFR), the duration, and area of backward flow in the pulmonary trunk, which

12 is a component of the vortex flow. The full width at half maximum (FWHM) of the

13 peak of the time course of VFR of backward flow was assessed to quantify the duration

14 of the vortical flow.

15

16 Results: Although overall flow patterns after BPA appeared to be the same as the one

17 before BPA, significant decreases in the FWHM, area, and VFR of the backward flow

18 after BPA were found (FWHM: before, $1.88 \times 10^{-1} \pm 1.51 \times 10^{-2}$ [cardiac cycle] vs. after, 
$191.65 \times 10^{-1} \pm 1.86 \times 10^{-2}$ [cardiac cycle]; area ratio: before, $2.67 \times 10^{-1} \pm 1.30 \times 10^{-2} \mathrm{vs.} \mathrm{after}$,

$202.38 \times 10^{-1} \pm 1.31 \times 10^{-2}$; VFR: before, $13.6 \pm 2.21[\mathrm{~mL} / \mathrm{s}]$ vs. after, $\left.11.3 \pm 2.36[\mathrm{~mL} / \mathrm{s}]\right)$.

21

22 Conclusion: BPA promoted significant decreases in the FWHM, area, and VFR of

23 backward flow in the pulmonary trunk, thereby facilitating efficient blood transport.

24 The tendencies for these changes were to be larger for cases where BPA more greatly

25 decreased the pressure. The results suggest that the FWHM, area, and VFR are useful

26 indicators for the noninvasive evaluation of the therapeutic effects of BPA.

27

28 Key words

29 4D-flow MRI; CTEPH; BPA; blood flow

30

31 


\section{Abbreviations}

33 Chronic thromboembolic pulmonary hypertension (CTEPH)

34 Pulmonary artery pressure (PAP)

35 Right heart catheterization (RHC)

36 Balloon pulmonary angioplasty (BPA)

37 Magnetic resonance (MR) imaging

38 Three-dimensional phase-contrast magnetic resonance imaging (4D-flow MRI)

39 Volume flow rate (VFR)

40 Electrocardiogram (ECG)

41 Pulmonary vascular resistance (PVR)

42 Right ventricular ejection fraction (RVEF)

43 Right ventricular cardiac index (RVCI)

$44 \quad$ Velocity encoding (VENC)

45 Full width at half maximum (FWHM)

46 Flow momentum index (FMI)

47 Reynolds number $(\mathrm{Re})$

48 Transtricuspid pressure gradient (TRPG) 
51 Chronic thromboembolic pulmonary hypertension (CTEPH) is characterized by the

52 presence of organized thrombi in the pulmonary arteries (PAs) [1]. Without appropriate

53 treatment, CTEPH has poor prognosis due to progressive right ventricular heart failure

54 [2]. Mean PA pressure (PAP) measured via right heart catheterization (RHC) is an

55 important metric in the diagnosis, prognosis, and response to therapy of this condition.

56 Pulmonary endarterectomy has been the standard treatment for CTEPH. However, it is

57 not suitable for patients whose arteriopathy is located in the distal vessels [3]. In this

58 regard, balloon pulmonary angioplasty (BPA) may be an alternative treatment for

59 patients with distal-type CTEPH or those not suited to surgery $[4,5]$.

60 Time-resolved, three-dimensional (3D), phase-contrast, magnetic resonance

61 imaging (4D-flow MRI) has been applied to analyze blood flow in the heart and large

62 vessels [6]. Its use allows not only the visualization of flow patterns but also analyses of

63 various fluid mechanical quantities, such as vorticity, helicity, and wall shear stress

$64[7,8,9]$.

65 Previous studies have implicated vortex flow in the PA as an abnormal blood

66 flow for patients with pulmonary hypertension [7,10,11]. Reiter et al. evaluated the

67 relative duration of the vortical flow in a cardiac cycle by viewing velocity fields and 
reported a strong correlation between the duration of vortical blood flow in the main PA

69 and mean PAP in patients with pulmonary hypertension [10]. This result strongly

70 suggests that vortical flow can be used as a marker of pulmonary hypertension.

71 However, visual assessment of blood flow is difficult, particularly when it has a

72 complex structure and variable momentum, and thus such assessment is prone to be

73 subjectivity and being dependent on the observer. In Reiter et al. (2014)[10], the

74 assessment was done by two readers with 12 years of experience in cardiac MRI. In

75 addition, visual assessment of blood flow with a complex 3D structure is

76 time-consuming because such a structure appears different depending on the viewing

77 angle; reportedly, the visual assessment of the vortical flow took between 15 and 25 min

78 per patient [10]. Therefore, a quantitative, objective evaluation of the vortex flow in the

79 PA that requires less effort is necessary for clinical application, such as evaluation of the

80 therapeutic effects and long-term monitoring of pulmonary hypertension.

81 In this study, we propose a more objective way of quantifying the vortical flow

82 in the PA with a focus on the backward flow component, a part of the vortex flow. The

83 duration, volume flow rate (VFR), and cross-sectional area of the backward flow was

84 evaluated from time-resolved, 3D velocity fields obtained by 4D-flow MRI. We also

85 assessed the structure of blood flow using various fluid mechanical indices that 
86 represent the flow complexity. The hemodynamics in the PA before and after BPA in

87 patients with CTEPH were compared to assess the efficacy of the proposed method.

88

89 


\section{Study population}

92 This study was approved by the local institutional review board and written informed

93 consent was obtained for each patient. In all, 28 CTEPH patients (7 men and 21 women;

94 mean age, 68 years; range, 50-83 years) were prospectively enrolled. Based on the

95 National Institute for Health and Care Excellence criteria [12], all patients were

96 diagnosed with CTEPH by medical history, physical examination, electrocardiogram

97 (ECG), chest X-ray, echocardiography, lung ventilation/perfusion scintigraphy, RHC,

98 and computed tomography angiography or PA angiography. The patients were treated

99 with the optimal medical therapy and several sessions of BPA (mean, $3.7 \pm 0.29$ ). In one

100 procedure, the target lesion was limited to 1 or 2 segments in one lobe to minimize the

101 complications from BPA. The sessions of BPA were repeated until the mean PAP

102 became less than $30[\mathrm{mmHg}]$ at a $4-8$ week interval [13]. RHC after interventions

103 showed decreases in mean PAP (before, $40 \pm 1.6[\mathrm{mmHg}]$ vs. after, $25 \pm 1.3[\mathrm{mmHg}]$ ).

104 The postoperative pressure decrease in mean PAP $(\Delta \mathrm{P})$ was $15 \pm 1.6[\mathrm{mmHg}]$ (range,

$1053-28$ [mmHg]; median, $14[\mathrm{mmHg}])$. RHC data before and after BPA is summarized in

106 Table 1. No patients manifested a severe complication, however, some patients had

107 blood sputum and mild or moderate hemoptysis; they were treated noninvasively. 


\section{Thoracic 4D-flow MRI}

110 Thoracic 4D-flow MRI examinations were performed in patients before and after

111 sessions of BPA (mean interval, $363 \pm 29.6$ [days]) using a $3.0 \mathrm{~T}$ scanner

112 (MAGNETOM Trio, A Tim System; Siemens Healthineers, Enlargen, Germany). The

113 MRI was performed with the following parameters: 3D phase-contrast MRI with

114 three-directional velocity encoding transverse acquisition; ECG gating; respiratory

115 gating; TR/TE, 42.6/2.54 [ms]; flip angle, $15\left[^{\circ}\right.$ ]; velocity encoding (VENC), 70 [cm/s],

116 spatial resolution, $2.4 \times 1.8 \times 3.5\left[\mathrm{~mm}^{3}\right]$; time resolution, $14-24$ [phases/cardiac cycle].

\section{Quantification of blood flow and vessel geometry}

119 Prototype post-processing software (4D-flow Demonstrator version 2.3; Siemens

120 Healthineers, Enlargen, Germany) was used to visualize the cardiovascular geometry

121 and blood flow patterns. Cross-sectional planes were set at the inlets of the pulmonary

122 trunk, right, and left main PAs to evaluate VFR, as shown in Fig. 1. In addition, 30

123 planes were set in the pulmonary trunk along the centerline to evaluate net VFR and

124 secondary flow in each plane. 
126 present) was quantified as the full width at half maximum (FWHM) of the peak of the

127 time course of VFR of backward flow (VFR), as depicted in Fig. 1. The FWHM does

128 not exactly represent the duration of vortex flow but dose quantify the length of time

129 that vortex flow exists with a high momentum, which is considered to have a relatively

130 large impact on pulmonary blood flow during a cardiac cycle.

131 The size of vortical flow was evaluated as the proportion of the area of the

132 backward flow present in each cross-section, and the time-averaged area ratio was

133 defined by

$134 \overline{\text { area ratio }}=\sum^{N_{\mathrm{p}}} A_{\mathrm{b}} / A / N_{\mathrm{p}}$

135 where $A_{\mathrm{b}}$ is the cross-sectional area occupied by the backward flow, $A$ is the entire

136 cross-sectional area, and $N_{\mathrm{p}}$ is the number of data acquisition phases of 4D-MRI (i.e.,

137 time resolution $14-24$ phases/cardiac cycle).

$139\left(v_{\mathrm{n}, \mathrm{f}}\right.$ and $\left.v_{\mathrm{n}, \mathrm{b}}\right)$, as shown in Fig. 1. The mean backward flow rate during a cardiac cycle is

$140 \quad \overline{\operatorname{VFR}_{\mathrm{b}}}=\sum^{N_{\mathrm{p}} \mathrm{VFR}_{\mathrm{b}}} / N_{\mathrm{p}}=\sum^{N_{\mathrm{p}} \int\left|\boldsymbol{v}_{n, \mathrm{~b}}\right| A_{\mathrm{b}}} / N_{\mathrm{p}}$

141 where $v_{\mathrm{n}, \mathrm{b}}$ is the normal component of the spatially averaged backward flow velocity.

143 Reynolds number (Re) defined as 
$144 \operatorname{Re}=u D / v$

145 where $u$ is a representative velocity, $D$ is the diameter of the blood vessel, and $v$ is the

146 kinematic viscosity. The normal component of the spatially averaged velocity in a

147 cross-sectional plane was used as the representative velocity. The kinetic viscosity of

148 blood was approximated by $v=3.3 \times 10^{-6}\left[\mathrm{~m}^{2 /} \mathrm{s}\right]$.

149 Flow complexity was assessed with various fluid mechanical indices, namely,

150 the flow momentum index (FMI), enstrophy density, and helicity density $\left(H_{\mathrm{d}}\right)$. The FMI

151 represents the relative strength of secondary flow [14] and is defined as the ratio

152 between the norms of the in-plane and a cross-sectional plane components of velocity

$153\left(\left|v_{\mathrm{s}}\right|\right.$ and $|v|$, respectively). Enstrophy density describes the strength of vorticity and is

154 defined as one half of the square of the vorticity [15]. $H_{\mathrm{d}}$ indicates the strength and

155 direction of rotation of the vorticity at a position and is defined as the inner product of

156 the velocity and vorticity [9].

The vortex structure of blood flow was characterized with the second invariant

158 of the velocity gradient tensor (Q) [16]. A region with positive Q-value represents the

159 existence of a vortex core, and thus Q value was evaluated to visualize the vortex 160 structure.

161 The geometric features of the PA, such as diameter, curvature, and bifurcation 
162 angle, were assessed. The mean diameter of the pulmonary trunk was calculated as

163 time-averaged mean diameter in the 30 planes. The vessel curvature is the reciprocal of

164 the radius of curvature that is equal to a circumradius of the triangle formed by a point

165 on the centerline and two neighboring points. 30 points were set in the centerline of the

166 pulmonary trunk, and the mean curvature was defined as the average of curvatures at

167 each point. The bifurcation angle was defined as the angle between the center of the

168 outlet of the pulmonary trunk and those of inlets of the right and left main PAs.

169 We assessed the parameters before and after BPA and explored the relationship

170 between the parameters and mean PAP obtained by RHC. The parameters were

171 calculated using in-house codes implemented with MATLAB 2018b software

172 (MathWorks, Natick, Massachusetts, USA). Data smoothing for velocity fields was

173 done with a $3 \times 3 \times 3$ median filter and 3D Gaussian filter $(\sigma=0.5)$.

\section{Statistical analyses}

176 Statistical parameters, such as the mean value, range, standard deviation, standard error,

177 and paired t-test results, were calculated using JMP pro version 14 software (SAS

178 Institute, Cary, North Carolina, USA). A $p$-value of less than 0.05 was considered to be

179 statistically significant. 
183 Volume flow rate and duration of backward flow in the main pulmonary artery

184 Figure 2 shows the FWHM, the area ratio, and $\overline{\mathrm{VFR}_{\mathrm{b}}}$ in the pulmonary trunk. The

185 values are the means obtained for the 30 cross-sectional planes in the pulmonary trunk.

186 Compared to their values before BPA, the FWHM, the area ratio, and $\overline{\mathrm{VFR}_{\mathrm{b}}}$ after BPA

187 all significantly changed: the FWHM shortened (before, $1.88 \times 10^{-1} \pm 1.51 \times 10^{-2}$

188 [cardiac cycle] vs. after, $1.65 \times 10^{-1} \pm 1.86 \times 10^{-2}$ [cardiac cycle], $\left.p=0.0429\right)$, the area

189 ratio decreased (before, $2.67 \times 10^{-1} \pm 1.30 \times 10^{-2}$ vs. after, $2.38 \times 10^{-1} \pm 1.31 \times 10^{-2}, p=$

190 0.0034), and the $\overline{\mathrm{VFR}_{\mathrm{b}}}$ decreased (before, $13.6 \pm 2.21[\mathrm{~mL} / \mathrm{s}]$ vs. after, $11.3 \pm 2.36$

$191[\mathrm{~mL} / \mathrm{s}], \quad p=0.009)$. The values of the perioperative ratios of FWHM

$192\left(=\mathrm{FWHM}_{\mathrm{after}} / \mathrm{FWHM}_{\mathrm{before}}\right), \quad$ area ratio $\left(=\left(A_{\mathrm{b}} / A\right)_{\mathrm{after}} /\left(A_{\mathrm{b}} / A\right)_{\text {before }}\right), \quad$ and $\quad \overline{\mathrm{VFR}_{\mathrm{b}}}$

$193\left(=\overline{\mathrm{VFR}_{\mathrm{b}, \mathrm{after}}} / \overline{\mathrm{VFR}_{\mathrm{b}, \text { before }}}\right)$ were $0.88 \pm 0.053,0.91 \pm 0.039$ and $0.91 \pm 0.099$,

194 respectively. There were no statistical correlations between $\triangle \mathrm{P}$ and FWHM, area ratio,

195 or $\overline{\mathrm{VFR}_{\mathrm{b}}}$.

196 We classified the cases into two groups with reference to the median $\Delta \mathrm{P}$ of 14

$197[\mathrm{mmHg}]:$ the large $(\Delta \mathrm{P} \geq 14[\mathrm{mmHg}], 15$ cases $)$ and small $(\Delta \mathrm{P}<14[\mathrm{mmHg}], 13$ cases $)$

198 decrease groups. The mean PAPs before BPA were $45 \pm 1.6[\mathrm{mmHg}]$ and $35 \pm 2.3$

$199[\mathrm{mmHg}]$ for the large and small decrease groups, respectively. The values after BPA 
200 were $23 \pm 1.6[\mathrm{mmHg}]$ and $28 \pm 2.0[\mathrm{mmHg}]$. Figure 3 shows the FWHW, area ratio,

201 and $\overline{\mathrm{VFR}_{\mathrm{b}}}$ for the two groups. All of the parameters significantly decreased in the large

202 decrease group (FWHM: before, $1.79 \times 10^{-1} \pm 1.61 \times 10^{-2}$ [cardiac cycle] vs. after, 1.44

$203 \times 10^{-1} \pm 1.54 \times 10^{-2}$ [cardiac cycle], $p=0.0064$; area ratio: before, $2.63 \times 10^{-1} \pm 1.27 \times$

$20410^{-2}$ vs. after, $2.25 \times 10^{-1} \pm 1.08 \times 10^{-2}, p=0.0058 ; \overline{V_{F R}}$ : before, $11.7 \pm 1.56[\mathrm{~mL} / \mathrm{s}] \mathrm{vs}$.

205 after, $7.9 \pm 9.99[\mathrm{~mL} / \mathrm{s}], p=0.0097)$. On the other hand, no statistically significant

206 postoperative differences were noted in the small decrease group (FWHM: before, 1.98

$207 \times 10^{-1} \pm 2.72 \times 10^{-2}$ [cardiac cycle] vs. after, $1.90 \times 10^{-1} \pm 3.56 \times 10^{-2}[$ cardiac cycle], $p$

$208=0.328$; area ratio: before, $2.72 \times 10^{-1} \pm 2.46 \times 10^{-2}$ vs. after, $2.52 \times 10^{-1} \pm 2.53 \times 10^{-2}$,

$209 p=0.114 ; \overline{V_{F R}}$ : before, $15.7 \pm 4.43[\mathrm{~mL} / \mathrm{s}]$ vs. after, $\left.15.1 \pm 4.64[\mathrm{~mL} / \mathrm{s}], p=0.373\right)$.

210

211 Volume flow rate at the inlets of the pulmonary trunk and bilateral main

212 pulmonary artery

213 Figure 4 shows the time-averaged VFR $(\overline{\mathrm{VFR}})$ at the inlet of the pulmonary trunk. As the

214 perioperative ratio was $1.34 \pm 0.186$, a statistically significant increase in the $\overline{V F R}$ after

215 BPA was discovered (before, $72.5 \pm 4.15[\mathrm{~mL} / \mathrm{s}]$ vs. after, $84.5 \pm 4.33[\mathrm{~mL} / \mathrm{s}], p=$

216 0.0148). VFRs increased at the inlets of the bilateral main PAs (right: before, $10.6 \pm$

$2171.56[\mathrm{~mL} / \mathrm{s}]$ vs. after, $14.4 \pm 2.07[\mathrm{~mL} / \mathrm{s}], p=0.0428$; left: before, $13.2 \pm 1.79[\mathrm{~mL} / \mathrm{s}] \mathrm{vs}$. 
218 after, $13.9 \pm 1.91[\mathrm{~mL} / \mathrm{s}], p=0.341)$. The split ratio of the flow between the right and

219 left main PAs $\left(=\overline{\mathrm{VFR}_{\text {right }}} /\left(\overline{\mathrm{VFR}_{\text {right }}}+\overline{\mathrm{VFR}_{\text {left }}}\right)\right)$ did not significantly change (before,

$22043 \pm 5.75 \%$ vs. after, $49 \pm 5.29 \%, p=0.262)$.

221

The $\overline{\text { VFR }}$ at the inlet of the pulmonary trunk significantly increased in the

222 large decrease group (before, $67.6 \pm 5.57[\mathrm{~mL} / \mathrm{s}]$ vs. after, $85.4 \pm 6.55[\mathrm{~mL} / \mathrm{s}], p=$

223 0.0173), whereas no significant change was observed in the small decrease group

224 (before, $78.2 \pm 6.08[\mathrm{~mL} / \mathrm{s}]$ vs. after, $83.4 \pm 5.73[\mathrm{~mL} / \mathrm{s}], p=0.118$ ). The perioperative

225 ratios were $1.54 \pm 0.34$ and $1.13 \pm 0.086$ in the large and small decrease groups,

226 respectively.

227

228 Blood flow structure and flow complexity

229 Figure 5 shows representative streamlines in late systole of two patients; one is in the

230 large decrease group whose mean PAP decreased from 48 to 20 [mmHg], and the other

231 is in the small decrease group whose mean PAP decreased from 47 to 40 [mmHg]. The

232 streamlines are colored according to the value of the streamwise velocity. The

233 perioperative changes in the streamlines did not show any notable difference between

234 the two cases. Before BPA, the flow was disturbed with vortices in the PAs (Fig. 5A and

235 C). Afterward, the flow disturbance in late systole was attenuated and streamwise 
236 velocity increased, although a vortical flow was still present in the pulmonary trunk (Fig.

$2375 \mathrm{~B}$ and $\mathrm{D})$.

Figure 6 shows isosurface plots of $H_{\mathrm{d}}$ and Q-value during late systole in those

239 same two patients as displayed in Fig. 5. The sign of $H_{\mathrm{d}}$ changes according to the

240 directions of the velocity and vorticity, with red (blue) indicating positive (negative)

241 values of $H_{\mathrm{d}}$. Regions of positive and negative $H_{\mathrm{d}}$ can be seen in the pulmonary trunk,

242 indicating that local blood flow was spinning in different directions. Considering the

243 perioperative change in distribution of positive and negative $H_{\mathrm{d}}$ regions, no notable

244 differences between the two cases were noted. Before BPA, positive and negative $H_{\mathrm{d}}$

245 regions were observed in the pulmonary trunk (Fig. 6A and C, left). Even after BPA,

246 positive and negative $H_{\mathrm{d}}$ regions were still observed, suggesting the existence of helical

247 flow (Fig. 6B and D, left).

248

The region of positive Q-value indicates the existence of a vortex core.

249 Likewise, there was no notable difference between the two cases, in terms of the

250 perioperative change in the distribution of the positive Q-value region (the large

251 decrease group, Fig. 6A and B, right; the small decrease group, Fig. 6C and D, right).

253 with BPA (FMI: before, $0.609 \pm 0.0128$ vs. after, $0.594 \pm 0.0122, p=0.917$; enstrophy 
254 density: before, $2.71 \times 10^{4} \pm 1.90 \times 10^{3}\left[1 / \mathrm{s}^{2}\right]$ vs. after, $2.95 \times 10^{4} \pm 3.94 \times 10^{3}\left[1 / \mathrm{s}^{2}\right], p$ $255=0.196)$

256

257 Reynolds number (Re) and vessel geometry

258 After BPA, peak and mean Re significantly increased (peak: before, $3580 \pm 158$ vs. after,

$2594120 \pm 214, p=0.0098$; mean: before, $888 \pm 49.5$ vs. after, $1110 \pm 60.8, p=0.0007)$.

260 The diameter of the pulmonary trunk significantly decreased (before, $34.8 \pm$

$2610.879[\mathrm{~mm}]$ vs. after, $33.4 \pm 0.828[\mathrm{~mm}], p=0.0009)$. The mean curvature of the

262 pulmonary trunk and bifurcation angle did not show any significant change (mean

263 curvature: before, $35.5 \pm 2.8\left[\mathrm{~m}^{-1}\right]$, vs after, $33.3 \pm 2.1\left[\mathrm{~m}^{-1}\right], p=0.47$; bifurcation angle:

264 before, $83 \pm 2.3\left[^{\circ}\right]$ vs after, $84 \pm 2.2\left[^{\circ}\right], p=0.59$ ).

265

266

267 
269 The major findings of the present study are two-folds. First, for the backward flow in

270 the pulmonary trunk, its duration, extent of its region, and its VFR all significantly

271 decreased after BPA. Second, the fluid mechanical indices representing the complexity

272 of blood flow did not show a significant change after BPA, despite an increase in Re

273 (i.e., the blood flow may now be disturbed more easily).

275 Perioperative changes in backward flow and whole pulmonary blood flow

276 A postoperative decrease in mean PAP by RHC is a major therapeutic effect $[5,17,18]$.

277 Pressure and velocity are closely associated in blood flow, and the pressure gradient can

278 be calculated from the velocity field using fluid mechanics. However, it is not possible

279 to calculate the absolute blood pressure solely from a velocity field. Previous studies

280 have investigated the relationship between mean PAP and velocity field [7,19,20,21].

281 Reiter et al. (2015)[10] observed vortices in the pulmonary trunk for patients with

282 pulmonary hypertension, and successfully demonstrated a strong correlation between

283 appearance time of vortices and mean PAP. Using the same technique, Ramos et al.

284 (2020)[21] demonstrated a correlation between mean PAP, estimated by the duration,

285 and the transtricuspid pressure gradient (TRPG). Because vortical flow in the PA has a 
3D structure and coexists with helical flow, it is difficult to accurately make a visual

287 assessment of changes in its size and duration. Therefore, a quantitative and objective

288 way to assess it is desired. For backward flow, the present results show decreases in

289 FWHM, extent of the region, and VFR after BPA. These changes were larger in cases

290 with larger pressure decreases. Therefore, these factors are useful indicators for

291 noninvasive evaluation of the therapeutic effects of BPA.

294 Perioperative changes in the complexity of blood flow

295 A flow pattern and its complexity are determined by vessel geometry and flow rate. In

296 terms of vessel geometry, the curvature from the pulmonary trunk and the bifurcation

297 angle did not show any significant change from BPA, while the vessel diameter

298 decreased. In fluid mechanics, Re is a key index to represent the nature of a flow, i.e.,

299 laminar or turbulent. In the present study, the mean Re increased, up to $\sim 1100$, after

300 BPA. This implies that the blood flow is still laminar, but a flow with a larger Re can be

301 disturbed more easily than the one with a smaller value [22]. Notwithstanding an

302 increase in Re, the streamlines after BPA demonstrate regression of the flow disturbance.

303 Moreover, the fluid mechanical indices that describe flow complexity, such as FMI and 
304 enstrophy density, did not show any significant changes after BPA. These perioperative

305 changes indicate that blood transport in the pulmonary trunk is facilitated by BPA.

307 Limitations

308 There were some limitations to this study. First, the VFR at the inlet of the pulmonary

309 trunk did not coincide with the sum of those at the inlets of the bilateral PAs, as shown

310 in Fig. 4. There are two factors associated with the inaccuracy of phase-contrast MRI:

311 the VENC setting and the flow-related dephasing in phase-contrast MRI. Concerinng

312 the former, the VENC might be set high to cope with the high velocity of the blood flow,

313 thus reducing the velocity sensitivity for any relatively slow flow. In the latter,

314 dephasing due to a disturbed flow can cause signal loss [23]. Second, the number of

315 patients was relatively small. Third, the follow-up 4D-flow MRI was performed only

316 once after BPA. After BPA, right ventricular reverse remodeling has been reported [17];

317 this would be associated with a change in the cardiovascular outcome in patients with

318 CTEPH. A long-term follow-up study could detect any additional changes in the PA

319 blood flow.

\section{Conclusions}


322 We developed an objective method of quantifying the vortex flow in the PA, focusing

323 on backward flow as a component of this vortex flow. This thoracic 4D-flow MRI study

324 of patients with CTEPH quantitatively demonstrated that the FWHM, the area ratio, and

325 VFR of the backward flow in the pulmonary trunk significantly decreased after BPA.

326 These changes tended to be larger in cases where $\Delta \mathrm{P}$ was larger. These results suggest

327 that these factors are useful indicators for noninvasive evaluation of the therapeutic

328 effects of BPA.

329

330 Acknowledgement

331 The authors are grateful to Tatsuo Nagasaka and Tomoyoshi Kimura for support with

332 4D-flow MRI scan, Shin Chibana for his support with post processing of image data,

333 Aurelien F. Stalder (Siemens Healthineer GmbH) for providing 4D-Flow Demonstrator,

334 and Yoshiaki Komori and Yuta Urushibata (Siemens Healthcare K.K.) for support with

335 acquisition of 4D-flow MRI.

336

337 Declarations

338 Conflict of interest

339 The authors have no conflict of interest to declare. 
$340 \quad$ Ethical approval

341 The present study was approved by the ethics committee of Tohoku University 342 (2014-1-875).

343

344

345

346

347 
349 1. D'Armini AM. Diagnostic advances and opportunities in chronic thromboembolic

350 pulmonary hypertension. Eur Respir Rev 2015;24(136):253-262. doi:10.1183/16000617.00000915

2. Dentali F, Donadini M, Gianni M, et al. Incidence of chronic pulmonary hypertension in patients with previous pulmonary embolism. Thromb Res 2009;124(3):256-258. doi:10.1016/j.thromres.2009.01.003

3. Yamada N. Beneficial Therapeutic Effects of Balloon Pulmonary Angioplasty on Biventricular Function in Patients With Chronic Thromboembolic Pulmonary Hypertension. Circ J 2016;80(6):1326-1327. doi:10.1253/circj.CJ-16-0418

4. Gopalan D, Delcroix M, Held M. Diagnosis of chronic thromboembolic pulmonary hypertension. Eur Respir Rev 2017;26(143):160108. Published 2017 Mar 15. doi:10.1183/16000617.0108-2016

5. Aoki $\mathrm{T}$, Sugimura $\mathrm{K}$, Tatebe $\mathrm{S}$, et al. Comprehensive evaluation of the effectiveness and safety of balloon pulmonary angioplasty for inoperable chronic thrombo-embolic pulmonary hypertension: long-term effects and procedure-related complications. Eur Heart J 2017;38:3152-9. 
Imaging 2012;36(5):1015-1036. doi:10.1002/jmri.23632.

367 7. Barker AJ, Roldán-Alzate A, Entezari P, et al. Four-dimensional flow assessment

368 of pulmonary artery flow and wall shear stress in adult pulmonary arterial 369 hypertension: results from two institutions. Magn Reson Med $370 \quad$ 2015;73(5):1904-1913. doi:10.1002/mrm.25326

371 8. Schäfer M, Barker AJ, Kheyfets V, et al. Helicity and Vorticity of Pulmonary 372 Arterial Flow in Patients With Pulmonary Hypertension: Quantitative Analysis of 373 Flow Formations. J Am Heart Assoc 2017;6(12):e007010. Published 2017 Dec 20. doi:10.1161/JAHA.117.007010

9. Kamada H, Ota H, Nakamura M, et al. Perioperative Hemodynamic Changes in the doi:10.1053/j.semtcvs.2019.07.006

10. Reiter G, Reiter U, Kovacs G, et al. Blood flow vortices along the main pulmonary artery measured with MR imaging for diagnosis of pulmonary hypertension. Radiology 2015;275(1):71-79. doi:10.1148/radiol.14140849

382 11. Ota H, Sugimura K, Miura $\mathrm{M}$ et al. Four-dimensional flow magnetic resonance imaging visualizes drastic change in vortex flow in the main pulmonary artery after 
percutaneous transluminal pulmonary angioplasty in a patient with chronic thromboembolic pulmonary hypertension. Eur Heart J 2015;36(25):1630. doi:10.1093/eurheartj/ehv054

12. Galiè N, Humbert M, Vachiery JL, et al. 2015 ESC/ERS Guidelines for the diagnosis and treatment of pulmonary hypertension: The Joint Task Force for the Diagnosis and Treatment of Pulmonary Hypertension of the European Society of Cardiology (ESC) and the European Respiratory Society (ERS): Endorsed by:

13. Sugimura K, Fukumoto Y, Satoh K, et al. Percutaneous transluminal pulmonary angioplasty markedly improves pulmonary hemodynamics and long-term Association for European Paediatric and Congenital Cardiology (AEPC), International Society for Heart and Lung Transplantation (ISHLT). Eur Heart J 2016;37(1):67-119. prognosis in patients with chronic thromboembolic pulmonary hypertension. Circ J disturbance by the fluid momentum index: Effect of the left ventricular systolic function on the hemodynamics in the aorta. Technol Health Care 2007;15(2):111120. 
402 15. Miura H, Enstrophy generation in a shock-dominated turbulence. J Phys Soc Jpn 1996;65(2):450-461. doi.org/10.1143/jpsj.65.450

404 16. Chen Q, Zhong Q, Meilan Qi, et al. Comparison of vortex identification criteria for planar velocity fields in wall turbulence. Phis Fluids 2015;27:085101.

17. Fukui S, Ogo T, Morita Y, et al. Right ventricular reverse remodelling after balloon pulmonary angioplasty. Eur Respir J 2014;43(5):1394-1402. doi:10.1183/09031936.00012914

18. Zoppellaro G, Badawy MR, Squizzato A, et al. Balloon Pulmonary Angioplasty in doi:10.1253/circj.CJ-19-0161

19. Han QJ, Witschey WR, Fang-Yen CM, et al. Altered Right Ventricular Kinetic Energy Work Density and Viscous Energy Dissipation in Patients with Pulmonary Arterial Hypertension: A Pilot Study Using 4D Flow MRI. PLoS One 2015;10(9):e0138365. Published 2015 Sep 29. doi:10.1371/journal.pone.0138365 arterial blood flow and wall shear stress in pulmonary arterial hypertension with 3D phase contrast magnetic resonance imaging. Springerplus 2016;5(1):1071. 
Published 2016 Jul 13. doi:10.1186/s40064-016-2755-7

421 21. Ramos JG, Fyrdahl A, Wieslander B, et al. Cardiovascular magnetic resonance 4D 422 flow analysis has a higher diagnostic yield than Doppler echocardiography for 423 detecting increased pulmonary artery pressure. BMC Med Imaging 2020;20(1):28. 424 Published 2020 Mar 6. doi:10.1186/s12880-020-00428-9

425 22. Spurk J, Aksel N, Fluid Mechanics second edition 2008, Springer, DOI 426 $10.1007 / 978-3-540-73537-3$

427

23. O'Brien KR, Cowan BR, Jain M, Stewart RA, Kerr AJ, Young AA. MRI phase

428 contrast velocity and flow errors in turbulent stenotic jets. J Magn Reson Imaging 2008;28(1):210-218. doi:10.1002/jmri.21395

430 
433 Figure 1. Assessment of blood flow in the pulmonary artery (PA). Planes were set at the

434 inlets of the pulmonary trunk and right and left main PAs to evaluate volume flow rate

435 (VFR). Moreover, 30 planes were set in the pulmonary trunk along the centerline to

436 evaluate net VFR and secondary flow in each plane. The centerline of the PAs was

437 defined as the normal direction of the plane. The duration of the vortical flow (the

438 length of cardiac phases with vortex present) was quantified as the full width at half

439 maximum (FWHM) of the peak of the time course of VFR of backward flow $\left(\overline{V_{F R}}\right)$.

440 The size of vortical flow was evaluated as the proportion of the area of the backward

441 flow present in each cross-section, and the time-averaged area ratio was defined by

$442 \overline{\text { area ratio }}=\sum^{N_{\mathrm{p}}} A_{\mathrm{b}} / A / N_{\mathrm{p}}$, where $A_{\mathrm{b}}$ is the cross-sectional area occupied by the

443 backward flow, $A$ is the entire cross-sectional area, and $N_{\mathrm{p}}$ is the number of data

444 acquisition phases of four-dimensional-flow magnetic resonance imaging (i.e., time

445 resolution $14-24$ phases/cardiac cycle). The velocity, $v$, in a cross-sectional plane may

446 be either forward or backward ( $v_{\mathrm{n}, \mathrm{f}}$ and $v_{\mathrm{n}, \mathrm{b}}$, respectively), as shown in Fig. 1. The mean

447 backward flow rate during a cardiac cycle is $\overline{\mathrm{VFR}_{\mathrm{b}}}=\sum^{N_{\mathrm{p}}} \mathrm{VFR}_{\mathrm{b}} / N_{\mathrm{p}}=$

$448 \sum^{N_{\mathrm{p}}} \int\left|v_{n, \mathrm{~b}}\right| A_{\mathrm{b}} / N_{\mathrm{p}}$, where $v_{\mathrm{n}, \mathrm{b}}$ is the normal component of the spatially averaged 
backward flow velocity.

450

451 Figure 2. Perioperative changes in the full width at half maximum (FWHM) of the peak

452 of the time course of volume flow rate of backward flow $\left(\overline{\mathrm{VFR}_{\mathrm{b}}}\right)$, area ratio between the

453 cross-sectional area occupied by the backward flow $\left(A_{\mathrm{b}}\right)$ and the entire cross-sectional

454 area $(A)$, and the time-averaged $\overline{\mathrm{VFR}_{\mathrm{b}}}$ during a cardiac cycle.

455

456 Figure 3. Perioperative changes in the full width at half maximum (FWHM) of the peak

457 of the time course of the volume flow rate of backward flow $\left(\overline{\mathrm{VFR}_{\mathrm{b}}}\right)$, area ratio between

458 the cross-sectional area occupied by the backward flow $\left(A_{\mathrm{b}}\right)$ and the entire

459 cross-sectional area $(A)$, and time-averaged $\overline{\mathrm{VFR}_{\mathrm{b}}}$ for two groups in reference to the

460 median decrease in mean pulmonary artery pressure, $\Delta \mathrm{P}=14[\mathrm{mmHg}]:$ the large $(\Delta \mathrm{P} \geq$

$46114[\mathrm{mmHg}], 15$ cases $)$ and small $(\Delta \mathrm{P}<14[\mathrm{mmHg}], 13$ cases $)$ decrease groups.

462

463 Figure 4. Perioperative changes in the volume flow rate (VFR) at the inlets of the (A)

464 the pulmonary trunk, and (B) right, and (C) left main pulmonary arteries (PAs).

465

466 Figure 5. Flow streamlines in late systole of two patients; one each from the large $(\Delta \mathrm{P} \geq$ 
$46714[\mathrm{mmHg}])((\mathrm{A})$ before and $(\mathrm{B})$ after BPA $)$ and small $(\Delta \mathrm{P}<14[\mathrm{mmHg}])$ decrease 468 groups ((C) before and (D) after BPA).

469

470 Figure 6. Isosurface plots of the helicity density $\left(H_{\mathrm{d}}\right)$ and second invariant of the

471 velocity gradient tensor (Q) during late systole in the same patients as those displayed in 472 Fig. 5. The plots of $H_{\mathrm{d}}$ and Q are shown in (A) before and (B) after BPA for the patient 473 in the large decrease group, and (C) before and (D) after BPA for the one in the small 474 decrease group, respectively. $\Delta \mathrm{P}$ : decrease in mean pulmonary artery pressure.

475

476

477

478 
480

481 Table 1: Right heart catheterization data before and after balloon pulmonary 482 angioplasty (BPA). Patients were classified into two groups with reference to the 483 median $\Delta \mathrm{P}$ of $14[\mathrm{mmHg}]:$ the large $(\Delta \mathrm{P} \geq 14[\mathrm{mmHg}], 15$ cases $)$ and small $(\Delta \mathrm{P}<14$ 484 [mmHg], 13 cases) decrease groups. PAP: pulmonary artery pressure, PVR: pulmonary 485 vascular resistance, RVEF: right ventricular ejection fraction, RVCI: right ventricular 486 cardiac index, $\Delta \mathrm{P}$ : decrease in mean pulmonary artery pressure.

487 


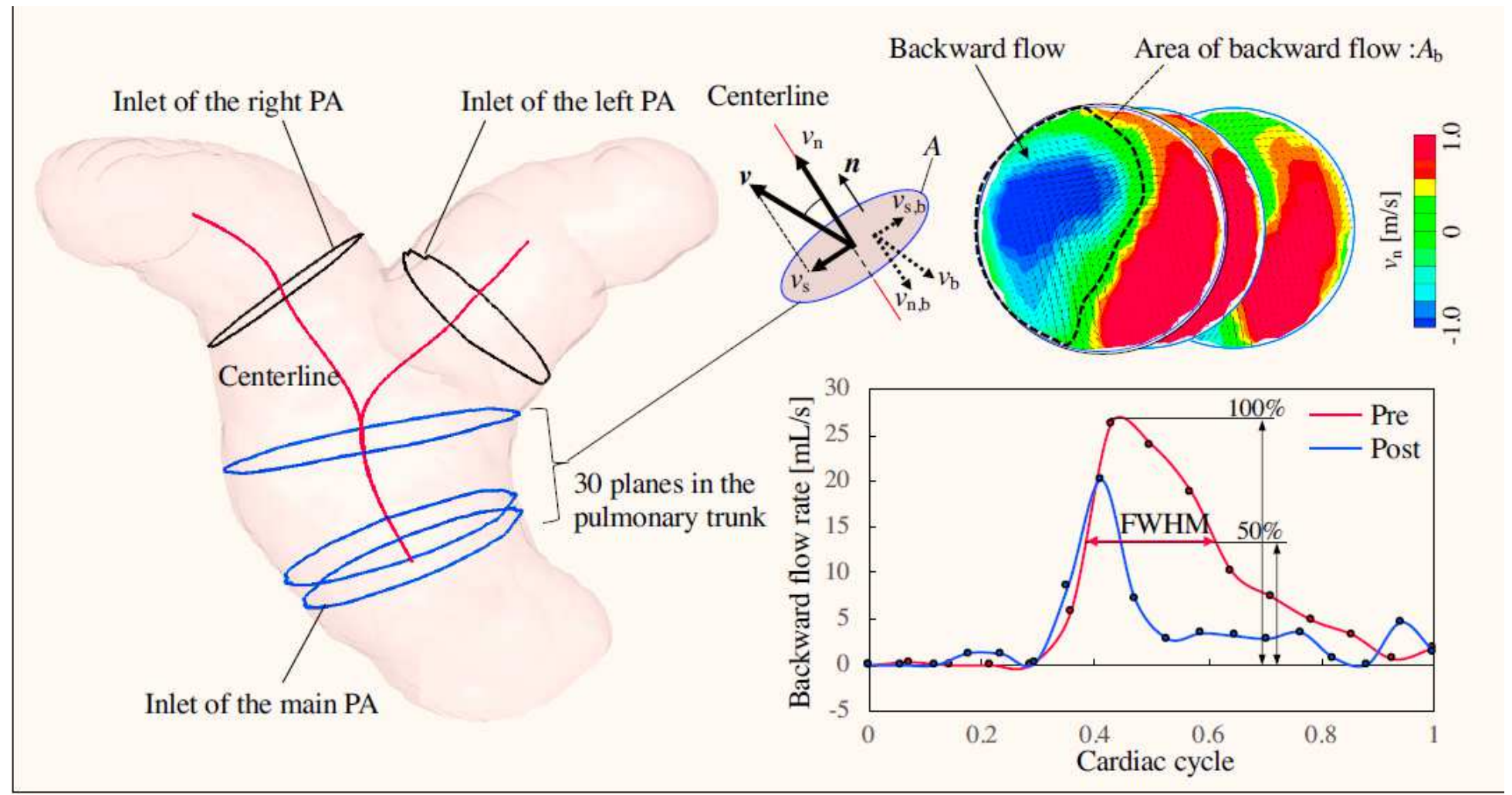

\section{Figure 1}

See the Manuscript Files section for the complete figure caption.
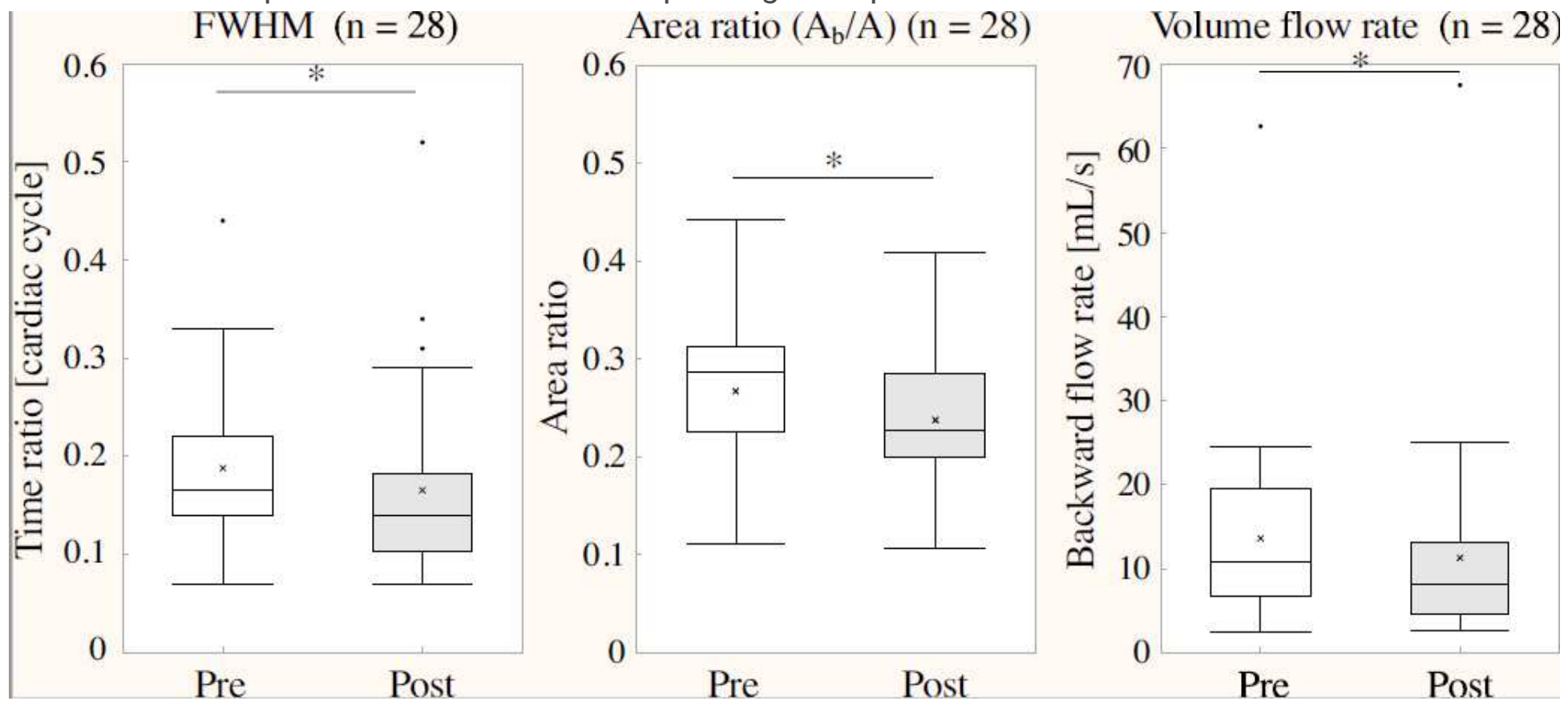

Figure 2

See the Manuscript Files section for the complete figure caption. 


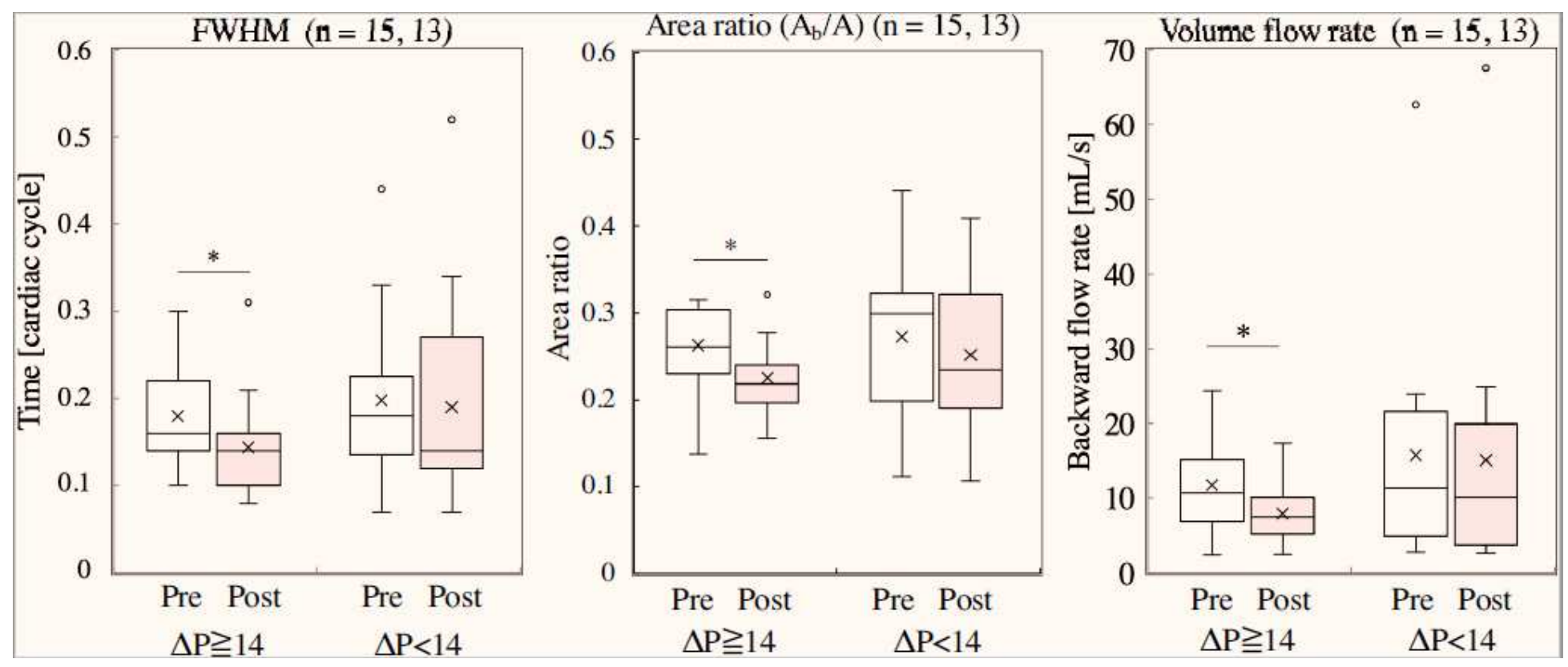

Figure 3

See the Manuscript Files section for the complete figure caption.

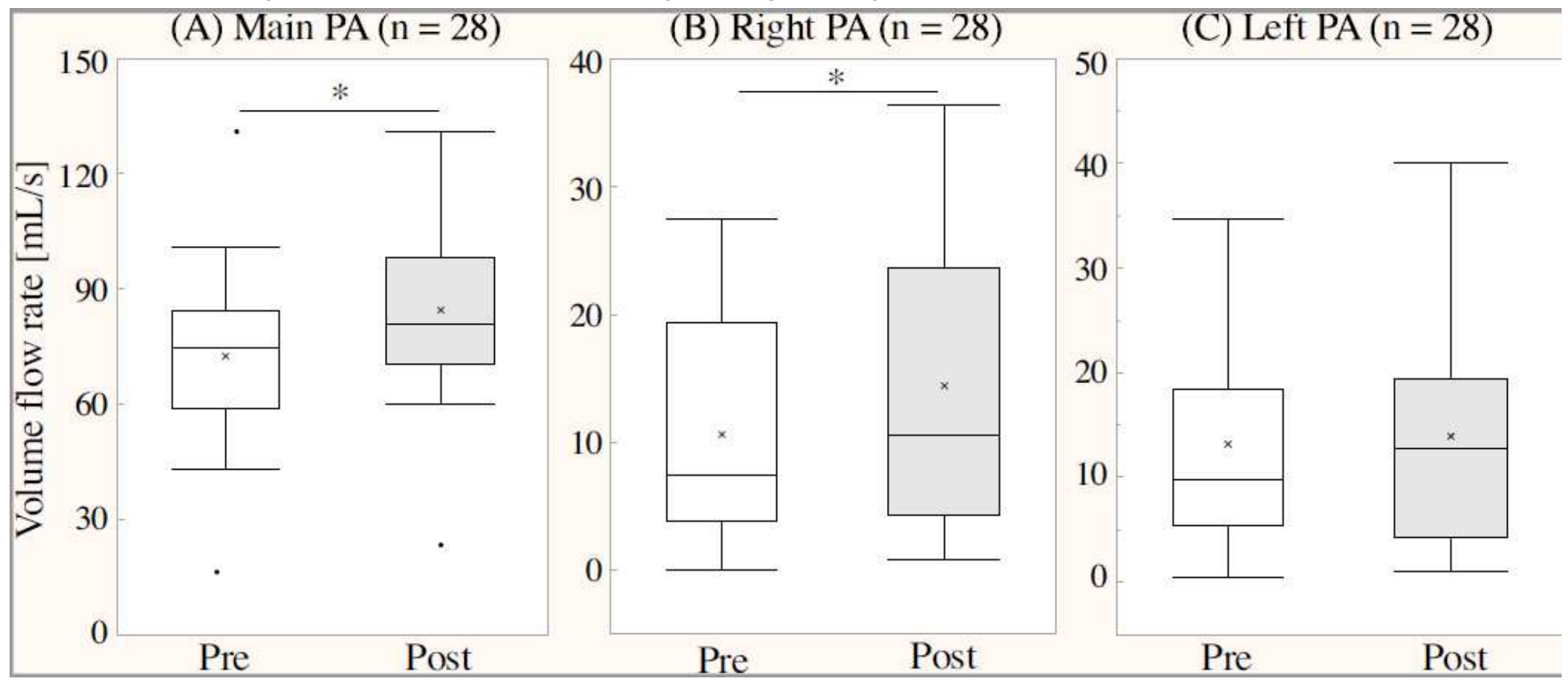

Figure 4

See the Manuscript Files section for the complete figure caption. 


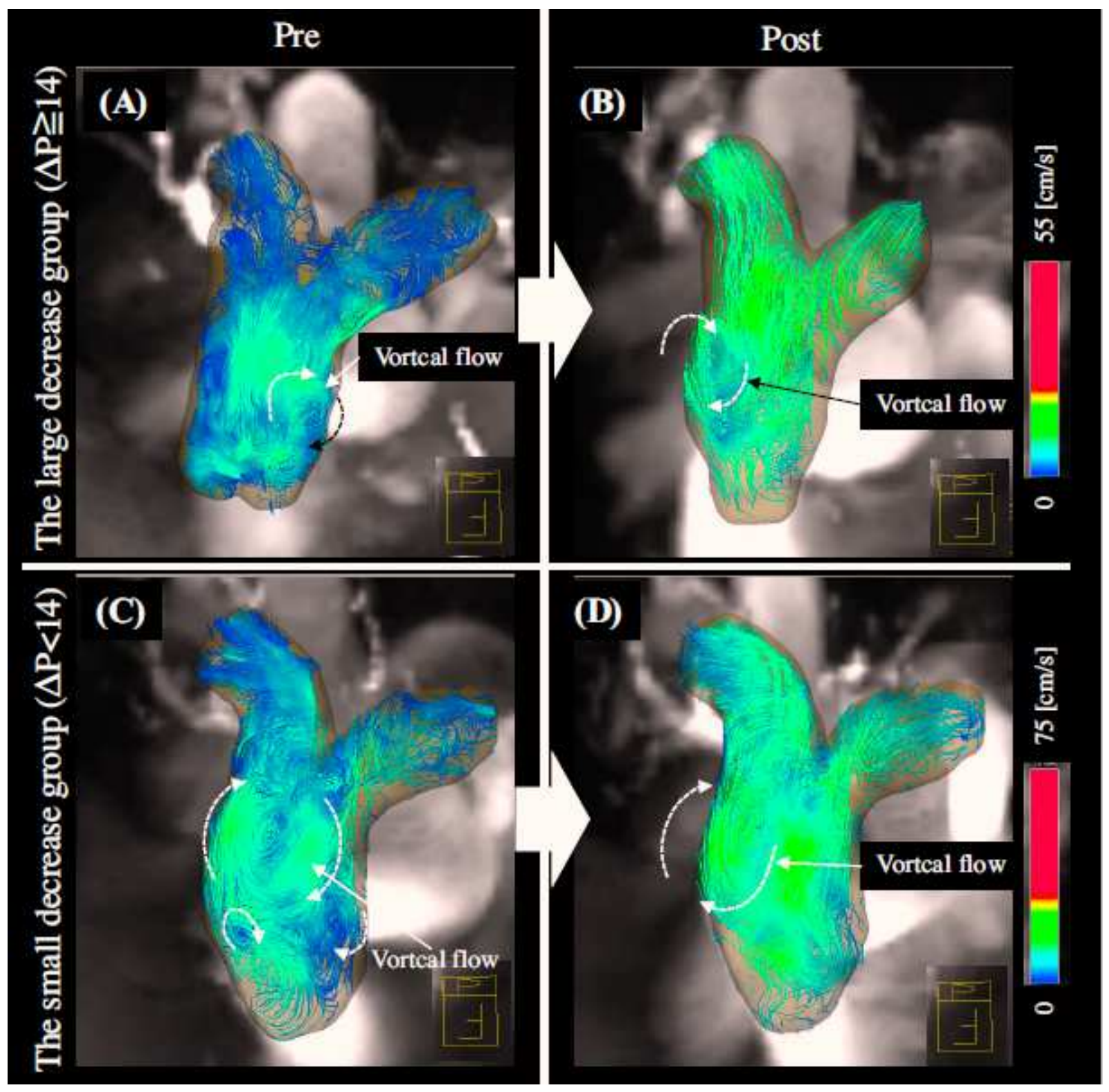

Figure 5

See the Manuscript Files section for the complete figure caption. 


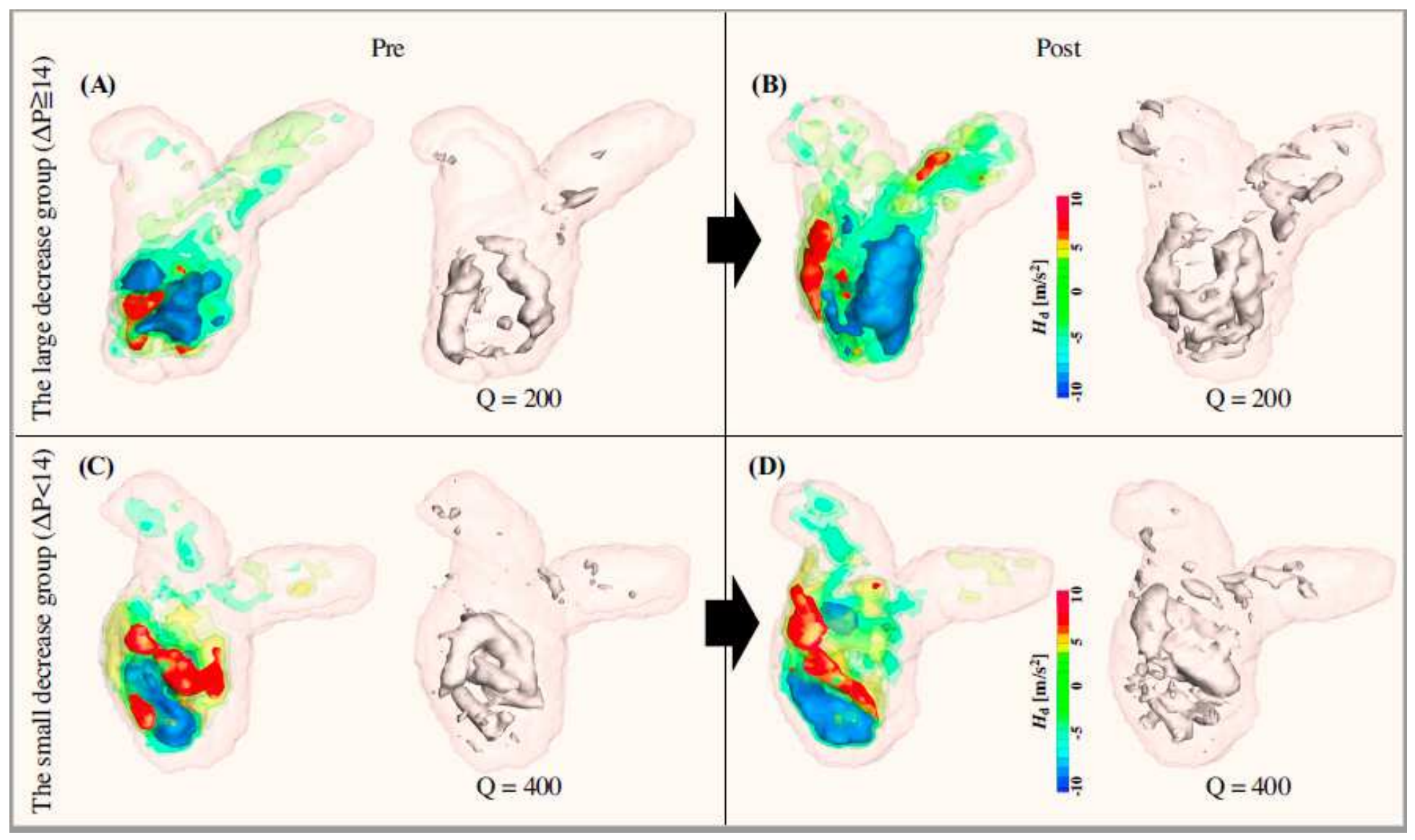

Figure 6

See the Manuscript Files section for the complete figure caption.

\section{Supplementary Files}

This is a list of supplementary files associated with this preprint. Click to download.

- Table1.eps 